TITLE:

\title{
A LIST OF BOTTOM ANIMALS COLLECTED BY A TRAWLER "KAIUN- MARU" OFF THE SOUTHWEST COAST OF KII PENINSULA
}

\author{
$\operatorname{AUTHOR}(\mathrm{S}):$
}

Utinomi, Huzio; Harada, Eiji

CITATION:

Utinomi, Huzio ...[et al]. A LIST OF BOTTOM ANIMALS COLLECTED BY A TRAWLER "KAIUNMARU" OFF THE SOUTHWEST COAST OF KII PENINSULA. PUBLICATIONS OF THE SETO MARINE BIOLOGICAL LABORATORY 1958, 6(3): 385-395

\section{ISSUE DATE:}

1958-06-20

URL:

http://hdl.handle.net/2433/174587

RIGHT: 


\title{
A LIST OF BOTTOM ANIMALS COLLECTED BY A TRAWLER "KAIUN-MARU" OFF THE SOUTHWEST COAST OF KII PENINSULA ${ }^{1 \text { ? }}$
}

\author{
HuzIo UTINOMI and EIJI HARADA \\ Seto Marine Biological Laboratory, Sirahama
}

With 1 Text-figure

\section{Introduction}

We had an opportunity of examining bottom animals that were taken by the trawl-net on the continental shelf off the southwest coast of Kii Peninsula, to afford some aspects of their composition and distribution.

The fishing ground of trawlers of Minabe, Wakayama Prefecture, is extending on the continental shelf 2 miles or more off along the coast from Hino-Misaki southward to Susami. The main object of the fishery is the fish, Taius tumifrons (TEmminck et Schlegel), with some others such as Zeus japonicus Cuvier et Valenciennes, Upeneus bensasi (Temminck et Schleger), or Hime japonica (Günther). But also various kinds of bottom animal are caught together with these fishes, to which little attention is paid by these fishermen and which the junior author collected on board for the examination. The materials were obtained on November 28th and December 8th, 1957, although precise positions of collection were decided and recorded on the latter occasion only. Of five stations of collection on December 8th, four were westwards of Seto-zaki, their depths being from 120 to 150 metres approximately and their natures of the ground coarse sand, sand or fine sand. The other station, having a depth of 80 metres approximately, was westwards of the mouth of Tanabe Bay, somewhat nearer the coast than the former four stations, its nature of the ground being fine sand. On the first occasion (November 28 th), the materials were taken mainly from the area around these former four stations.

The bottom animals thus collected, though only on two occasions, consist of 188 species belonging to 12 phyla, in which the fishes, mollusks and coelenterates were most abundant. The invertebrates were more abundant on deeper stations, especially the coelenterates on those of the coarse sandy or sandy bottom. Endopachys japonicum YABE et EGUCHI was found very abundantly at the deepest station (St. 4) of the

1) Contributions from the Seto Marine Biological Laboratory, No. 312.

Publ. Seto Mar. Biol. Lab., VI (3), 1958. (Article 20) 
coarse sandy bottom exclusively. Of the mollusks, Guildfordia triumphans (PHILIPPI), Xenophora pallidula (REEve) and Apollon (Biplex) peca (PERRY) were dominant all over deeper stations. With regard to the fish, there appears to be distinct difference between their compositions from deeper stations and from a shallower station.

\begin{tabular}{|c|c|c|c|c|c|}
\hline \multirow{2}{*}{ Date } & \multirow{2}{*}{ Station } & \multicolumn{2}{|c|}{ Position } & \multirow{2}{*}{$\begin{array}{c}\text { Approx. } \\
\text { Depth } \\
\text { (metre) }\end{array}$} & \multirow{2}{*}{ Substratum } \\
\hline & & Long. (E) & Lat. (N) & & \\
\hline Nov. 28,1957 & \multicolumn{3}{|c|}{ Area neighbouring St. 1-4 } & $80-150$ & fine-coarse sand \\
\hline Dec. 8,1957 & 1 & $135^{\circ} 12.2^{\prime}$ & $33^{\circ} 40.0^{\prime}$ & 120 & fine sand \\
\hline$"$ & 2 & $135^{\circ} 10.2^{\prime}$ & $33^{\circ} 41.1^{\prime}$ & 120 & sand \\
\hline$”$ & 3 & $135^{\circ} 09.7^{\prime}$ & $33^{\circ} 39.7^{\prime}$ & 140 & sand \\
\hline$"$ & 4 & $135^{\circ} 09.4^{\prime}$ & $33^{\circ} 40.0^{\prime}$ & 150 & coarse sand \\
\hline " & 5 & $135^{\circ} 16.0^{\prime}$ & $33^{\circ} 42.2^{\prime}$ & 80 & fine sand \\
\hline
\end{tabular}

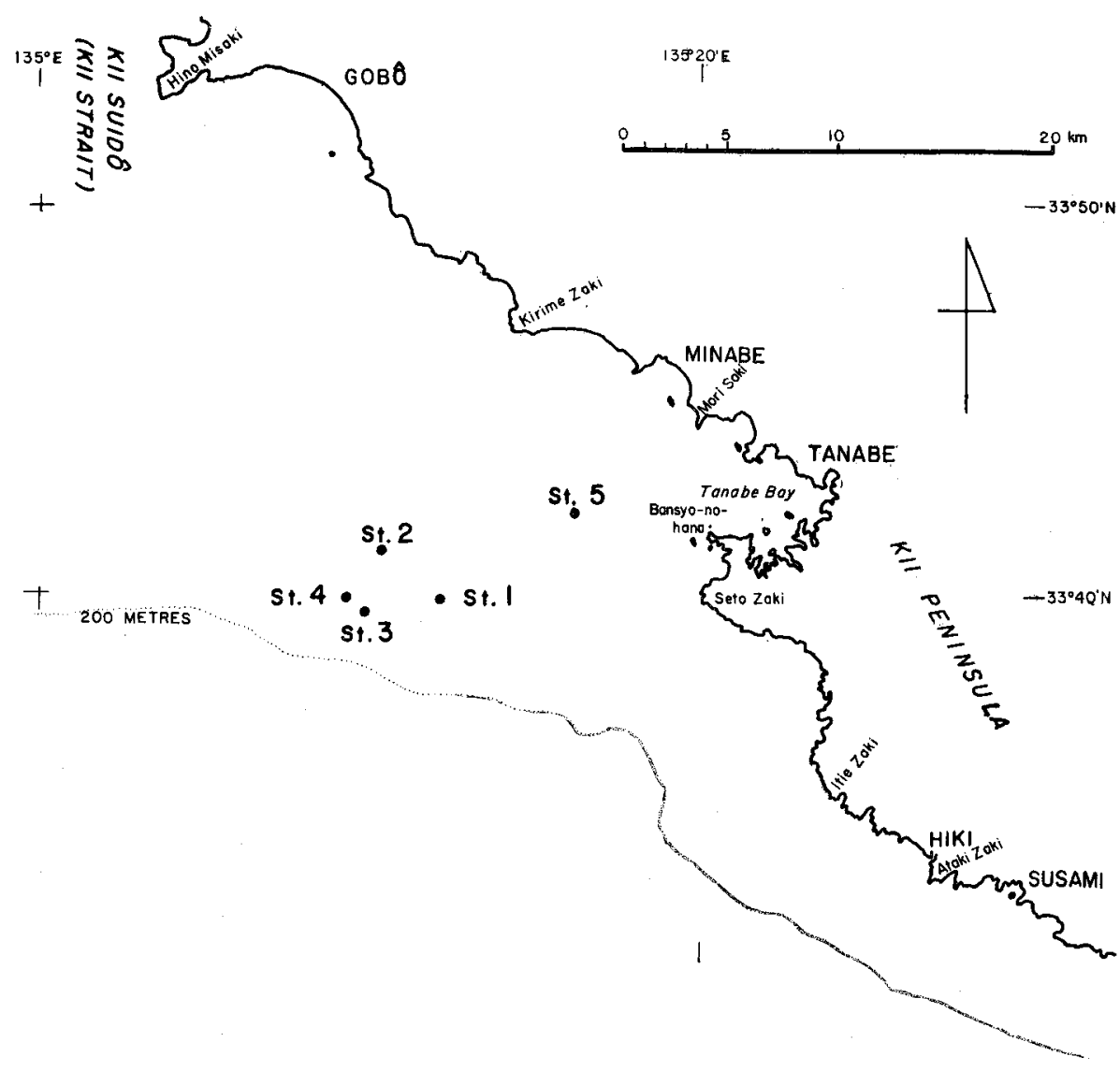

Fig. 1. Chart showing the positions of collection ( ) by trawling on December 8 th, 1957. 


\section{Acknowledgments}

We wish to thank Mr. Ichiro YAGURA, Captain of the trawler "Kaiun-maru" and his crew for allowing us to make collection on board and affording facilities and help for it. Identifications of most of the invertebrates, though tentatively, were made by the senior author and those of fishes were made by the junior author. But Dr. Sadayoshi Mryake kindly identified the anomuran crustaceans and Dr. Itsuo Kubo the caridean crustaceans. Dr. Takasi Tokıok a was also responsible for the tunicates and Mr. Torao Yamamoto was consulted for the mollusks. We are much obliged for these aids.

\section{Phylum PROTOZOA \\ Class Rhizopoda \\ Order Foraminifera}

Homotrema rubrum (LAMARCK) アカスナヨ

St. 1

\section{Phylum PORIFERA \\ Class Hexactinellida}

Euplectella oweni HeRKLots et MARSHALL オウエンカイロウドウタy St. $2,3,4$ ?Polylophus philippinensis ScHulteE

Class Demospongiae

Stelleta sp.

Gen.? spec.?

St. 2

\section{Phylum COELENTERATA}

\section{Class Hydrozoa}

Order Hydroida

Gonothyrea bicuspidata (CLARKE) フタエキザミ

Clytia linearis THORNELy エダウミコップ

Symplectoscyphus tricuspidatus (ALDER) トグウミンハ:

Amphisbetia furcata (TRASK) ヒメウミカビ

Sertularella sp.

Synthecium campylocarpum AlLMAN ッッウミンバ

Gen.? spec.?

\section{Class Anthozoa \\ Subclass Alcyonaria \\ Order Stolonifera}

Sarcodictyon gotoi (Oкuво) comb. nov. 
Order Alcyonacea

Bellonella rigida PÜTTER ボウウミイチゴ

St. $1,2,3,4$

B. dofleini (KÜKENTHAL) エダウミイチゴ

St. 1, 2, 3, 4

B. macrospina (KÜKENTHAL) オオトゲウミイチゴ

St. 1, 2, 3, 4

Daniela koreni $\mathrm{KoCH}$

Kochella studeri (KocH)

Anthomastus granulosus KüKENTHAL エナガウミテングタケ(新称)

St. $1,2,3,4$

Minabea ozakii UTinomI ミナベト少为

St. $2,3,4$

Dendronephthya acaulis KÜKENTHAL コデマリトゲトサカ

St. $1,2,3,4,5$

Siphonogorgia dipsacea (WRIGHT et STUDER) アカパナクダヤギ

St. 2

Order Gorgonacea

Euplexaura? attenuata (NUTTING)

Filigella mitsukurii KInoshitA イトヤギ(新称)

St. 1

Paramuriceidae (gen.? spec.?)

Echinomuricea? sp.

\section{Order Pennatulacea}

Echinoptilum macintoshi HUBRECHT トグサボテン

Funiculina quadrangularis (PALLAS) ムテウミエラ

Leioptilus fimbriatus (HERKLOTS) ウミエす

St. $1,2,4$

\section{Subclass Zoantharia}

\section{Order Actiniaria}

? Phyllactis striata WASSILIEFF

Actiniidae (gen.? spec.?)

St. 2,4

Halcampella maxima R. HERTwIG ナスビイソギンチヤク

St. 1,4

\section{Order Scleractinia}

Flabellum pavoninum paripavoninum ALCOCK センスガイ

St. 2,4

$F$. distinctum Milne Edwards et HaIme

St. $1,2,4$

F. rubrum (QUOY et GAIMARD)

$F$. deludens v. MARENZELLER

Stephanocyathus (Odontocyathus) spiniger (v. MARENZELLER) コウロサンゴ（新称)

Heterocyathus aequicostatus M. EDWARDs et HAIME

Bored by a commensal sipunculid, Aspidosiphon sp.

Caryophyllia sp.

Endopachys japonicum YABE et EGUCHI

St. 4

Dendrophyllia fistula (ALCOCK)

St. 2

D. arbuscula VAN DER HORST

St. 4 
Balanophyllia gigas MoselEy

St. 2, 3, 4

Trochocyathus (Thecocyathus) hanzawai YABE et EGUCHI

\section{Phylum ANNELIDA \\ Class Polychaeta \\ Order Polychaeta}

Onuphis willemoesic (McInTosH) カナブツイソメ

St. 1

Peculiar dwelling tube only.

?? Mesochaetopterus sp. Dwelling tubes only.

Pomatostegus latiscapus MARENzeller

St. $1,3,4$

? Hydroides norvegicus (GUNnerus) カ少市カザシ

Lumbrineris latreilli (Audouin et MiLne Edwards) ギボンイソタ

St. 2

Stylarioides parmatus GRUBE

Glycera opisthobranchiata MARENZELLER マイヅルキロリ

Dwelling tube of undet. Polychaeta

St. 5

Order Myzostomida

Myzostomum ambiguum GRAFF ツノスイクチムシ

\section{Phylum SIPUNCULOIDEA}

Sipunculus nudus LINNÉ スシホシムシ

\section{Phylum ARTHROPODA \\ Class Pycnogonida}

Ascorhynchus auchenicum (SLATER) トゲトツクリウミグモ（新称）

\section{Class Crustacea}

Order Cirripedia

Balanus (Chirona) tenuis HOEK シロフシッボ(新称)

St. 2

Sacculina sp.

Parasitic on a crab, Actumnus squamosus.

Order Isopoda

Gnathia sp.

Ianira chuni (THIELEMANN)

\section{Order Amphipoda}

Lilljeborgia brevicornis (BRUZELIUS)

Melita aff. gladiosa BATE 
Maera sp.

Neopleustes boecki (HANSEN)

Paramphithoe sp.

One sp. undet.

\section{Order Decapoda}

\section{Suborder Natantia}

Parapenaeus investigatoris AlCoCk et ANDERSON

Parapandalus spinipes (BATE) オキノンテエビ

St. $1,2,3$

St. 1

Solenocera sp.

Synalpheus sp.

Plesionika sp.

Spongicola venusta DE HAAN ドウケツエビ

Lodged commensally in the gastral cavity of a sponge,

Euplectella oweni.

St. $2,3,4$

\section{Suborder Reptantia}

Tribe Palinura

Ibacus ciliatus (VON SIEBOLD) ウチワエビ

St. $1,2,3$

Tribe Anomura

Dardanus arrosor (HERBST) ヨコミゾヤドカり

St. 1, 2, 3, 4

Pagurus ophthalmicus (OrTmanN)

Pagurus sp.

Paguristes palythophilius ORTMANN

St. 1, 2

Parapagurus diogenes (WHITELEGGE)

Galathea pubescens STIMPSON ケブカガラデ

G. subsquamata STrMPSON

G. elegans WHITE コマチガラテア

Uroptychus scandens BENEDICT トグエビモドキ

Lodged commensally in the ventral groove of a sea-pen,

Leioptilus fimbriatus.

St. $1,2,4$

Munida japonica japonica STIMPSON ヤマトムニダ

Cervimunida princeps BENEDICT ツノガムニダ

St. 2

Tribe Brachyura

Homola orientalis HENDERSON トウヨウホたラ

Latreillia phalangium DE HAAN ミズヒキガニ

Latreillopsis bispinosa HENDERSON トグミズヒキガニ

St. 2, 3, 4

Pleistacantha sancti-johannis MIERS ハリセンボン

St. 4

Leptomithrax edwardsi (DE HAAN) コンマガ二

St. 3

St. 1,2 
Charybdis bimaculata (MTERS) フタホンインガ二

C. miles DE HAAN アカインガニ

St. 5

Actumnus squamosus DE HAAN イボデニ

Some specimens carry Sacculina sp.

St. $1,2,3,4$

\section{Phylum MOLLUSCA \\ Class Pelecypoda \\ Order Anisomyaria}

*Pecten (Notovola) albicans (SCHRÖTER) イタヤガイ

Cryptopecten vesiculosus (DUNKER) ヒヨクガイ

St. 4

Ostrea sp.

*Meiocardia tetragona (ADAMS et REEVE) コウホホガイ

Order Eulamellibranchia

Clavagella (Stirpulina) ramosa DUNKER ハマコウ

\section{Class Gastropoda}

\section{Order Archaeogastropoda}

Calliostoma (Tristichotrochus) aculeatum SowERBY トグエビス

St. 4

C. haliarchus (MELvill) ヒラコマ

St. 1

Guildfordia triumphans (Phul.IPPI) リンボウガイ

St. 1, 2, 3, 4

\section{Order Mesogastropoda}

Architectonica maxima (PHILIPPI) クルマガイ

*? Siliquaria sp.

St. $1,2,3$

Vermiculariidae (gen.? spec.?)

*Onustus exutus (REEvE) キヌガサガイ

Xenophora pallidula (REEvE) クマ少ガイ

St. $1,2,3,4$

Neverita (Glossaulax) didyma (RöDING) ツメタガイ

*Semicassis japonica (REEvE) ウホウランマ

Apollon (Biplex) peca (PERRY) マツカワガイ

St. $1,2,3,4$

Tritonalia sauliae (REEve) ボウシユウボ

St. 1

*Bursa (Bufonariella) ranellvides (REEvE) コナルトボす

\section{Order Neogastropoda}

Murex (Siratus) pliciferoides KuRODA ハツキガイ

St. 1,2

Ceratostoma (Ocenebra) eurypteron (ADAMS et REEvE) ヨウラクヒレガイ

Latiaxis (Tolema) japonicus (DUNKER) テンニヨノカムリ

St. 2

L. pagodus (A. ADAMS) ヒメカセン

St. 4

\footnotetext{
* Dead shells only.
} 
Fusinus simplex (SMITH) ンロヒメナガニン

St. 1, 2

*F. forceps (PERRY) イトマキナガニン

*Granulifusus niponicus (SMIтH) アラレナ方二ン

Gemmula granosa (Helbling) シュズカケクダマキ

*Nassarius (Zeuxis) caelatus (A. ADAMS) 八广ムシロ

*Asprella (Endemoconus) sieboldi (REEVE) アコメカ十

A. (Conasprella) cancellata (BRUGUIERE) ヒシイモサイ

Order Nudibranchia

Nudibranchia (gen. ? spec.?)

\section{Class Cephalopoda}

Order Decapoda

Sepia (Sepia) esculenta HoYLE コウイカ

St. 1, 2, 3, 4

Phylum BRYOZOA

Order Cheilostomata

Adeona japonica (ORTMANN) ツノコケムシ

\section{Phylum BRACHIOPODA}

Order Telotremata

Laqueus rubellus (SOWERBY) ホウズキチヨウテン

\section{Phylum ECHINODERMATA \\ Class Crinoidea \\ Order Articulata}

Comantheria grandicalyx (CARPENTER) オオバンウミンダ Comatulida (gen.? spec.?)

St. 3,4

St. 4

Class Asteroidea

Order Phanerozonia

Ctenopleura ludwigi (DE LORIOL)

Luidia maculata MüLLER et TroscheL ヤツデスナヒトデ

St. 2

Pteraster tesselatus Ives

St. 1

\section{Order Spinulosa}

Henricia pachyderma HAYASHI

Hymenaster glaucus SLADEN マクヒトデ

St. 4 


\section{Class Ophiuroidea}

Order Euryalae

Gorgonocephalus sp.

$$
\text { Order Ophiurae }
$$

Stegophiura sp.

2 other spp. undet.

$$
\begin{aligned}
& \text { Class Echinoidea } \\
& \text { Order Cidaroidea }
\end{aligned}
$$

Stereocidaris grandis (DöDERLEIN) フトザオウニ

St. 1,4

Goniocidaris (Petalocidaris) biserialis (DÖDERLEIN) トダザオウニ

St. 1, 4

\section{Order Camarodonta}

Temnopleurus (Toreumatica) apodus (A. AgAssiz et H. L. CLARK) コッブサンンヨウウニ

Chaetodiadema japonicum MORTENSEN ヤワラガゼ

St. 1, 4

Order Clypeastroida

Clypeaster virescens DöDERLEIN オオタコノマクラ

St. 3

Class Holothurioidea

Order Aspidochirota

Stichopus sp.

St. 1, 4

Holothuria sp.

St. 3

\section{Phylum PROCHORDATA \\ Class Urochordata \\ Subclass Ascidiacea}

? Syndiazona grandis OKA, juv. ボウズボヤ

St. 1

Homoeodistoma longigona ToKIoK. (MS.)

St. 1

Subclass Pyrosomata

Pyrosoma spinosum (HERdMAN).

St. 1

P. atlanticum atlanticum PÉRON ヒカリボヤ

St. 1

\section{Phylum VERTEBRATA \\ Class Chondrichthyes \\ Order Lamnida}

Mustelus griseus PIETSCHMANN シロザメ

St. 1, 4 


\section{Order Rajida}

Rhinobatus schlegeli MÜLLER et HENLE サカダザ

Raja kenojei MÜLLER et HENLE ガンギエイ

St. 4

Dasyatis akajei (MüLLER et HENLE) アカエイ

St. 3,5

\section{Class Osteichthyes}

Order Myctophida

Hime japonica (GỨNTHER) ヒメ

St. $1,2,3,5$

Saurida undosquamis (RICHARDSON) マエy

St. 3,4

Synodontidae (gen.? spec.?)

St. $1,2,3,4$

Order Anguillida

Muraenesox cinereus (FORSKÅL) 八‡

St. $1,2,3$

\section{Order Syngnathida}

Fistularia villosa KLUNZINGER アオヤガラ

\section{Order Berycida}

Monocentris japonicus (HouTTUYN) マッカサウォ

\section{Order Zeida}

Zeus japonicus Cuvier et VAlenciennes

St. $1,2,3,4$

\section{Order Percida}

?Sphyraena pinguis GüNTHER アカカマス

Trachurus japonicus (TEMmINCK et SCHLEGEL) マアシ

St. 1,2

Caranx equula Temminck et Schlegel カイワリ

St. 5

Leiognathus rivulatus (TEMminck et SChlegel) オキヒイラギ

St. 5

Upeneus bensasi (Temminck et Schlegel) ヒメシ

Branchiostegus japonicus japonicus (HouTTuYN) アカアマダイ

St. $1,2,3,4,5$

Apogon kiensis Jordan et SNyder テッポウイシモチ

St. $1,3,5$

Pseudopriacanthus niphonius (CUvIER) クルマダイ

St. 5

Acropoma japonicum GÜNTHER ホ夕ルシャコ

St. 1, 2, 3, 4

St. 5

Epinephelus septemfasciatus (THUNBERG) マ八夕

St. 4

Nemipterus bathybus SNYDER ソコイトヨり

St. 5

Taius tumifrons (Temminck et SCHLEGEL) キダイ

St. $1,2,3,4$

Scolopsis inerme (Temminck et SCHLEGEL) タマガンラ

St. 5

Neopercis sexfasciata (TEMmINCK et SCHLEGEL) クラカケトラギス

St. $1,2,3,4,5$

N. multifasciata (DöDERLEIN) オキトシギス

St. $1,2,3,4$

Uranoscopus japonicus HoUTTUYN ミシマオコゼ

St. 1

Calliurichthys japonicus (HoutTuYN) ヨメゴチ

St. $1,2,3,4$ 
Microcanthus strigatus (Cuvier et VALENCIENNES) カゴカキダイ

Antigonia rubescens (GÜNTHER) ベニヒシダイ

St. 5

St. 1, 2, 3, 4

\section{Order Cottida}

Scorpaena izensis JoRDAN et STARKS イズカサゴ

Pterois lunulata Temminck et Schlegel ミノカサゴ

St. $1,2,3,4$

Scorpaenidae (gen.? spec.?)

St. 2

St. 3

Inimicus japonicus (CUVIER et VALENCIENNES) オニオコゼ

?Lepidotrigla abyssalis JORDAN et STARKS ソコカナガンラ

St. 1, 2, 3, 4

?L. japonica (BLEEKER) トグカナガンラ

St. 1, 2, 3, 4

Order Tetraodontida

Kentrocapros aculeatus (HouTTuYN) イトマキフグ

St. $1,2,3$

Order Pleuronectida

Bothidae (gen.? spec.?)

St. $1,2,3,4,5$

Pleuronichthys cornutus (TEMminck et SCHLEGEL) メイタガレイ

St. $1,2,5$

\section{Order Lophiida}

Lophiomus setigerus (VAHL) アンコウ

St. 1

Antennarius laterallis TANAKA オオモンイザりウ才

St. 1

Halieutaea stellata (VAHL) アカグツ

St. 2 